\title{
Nuclear ErbB2 represses DEPTOR transcription to inhibit autophagy in breast cancer cells
}

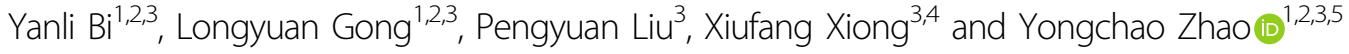

\begin{abstract}
ErbB2, a classical receptor tyrosine kinase, is frequently overexpressed in breast cancer cells. Although the role of ErbB2 in the transmission of extracellular signals to intracellular matrix has been widely studied, the functions of nuclear ErbB2 remain largely elusive. Here, we report a novel function of nuclear ErbB2 in repressing the transcription of DEPTOR, a direct inhibitor of mTOR. Nuclear ErbB2 directly binds to the consensus binding sequence in the DEPTOR promoter to repress its transcription. The kinase activity of ErbB2 is required for its nuclear translocation and transcriptional repression of DEPTOR. Moreover, the repressed DEPTOR by nuclear ErbB2 inhibits the induction of autophagy by activating mTORC1. Thus, our study reveals a novel mechanism for autophagy regulation by functional ErbB2, which translocates to the nucleus and acts as a transcriptional regulator to suppresS DEPTOR transcription, leading to activation of the PI3K/AKT/mTOR pathway to inhibit autophagy.
\end{abstract}

\section{Introduction}

The mammalian target of rapamycin (mTOR), an evolutionarily conserved serine/threonine protein kinase, serves as a central regulator of cell growth, proliferation, survival, and autophagy, and is frequently activated in many human cancers ${ }^{1}$. DEPTOR (DEP-domain containing mTOR-interacting protein), a naturally occurring inhibitor of mTOR, directly binds to mTOR and suppresses the kinase activity of mTOR complex 1 (mTORC1) and 2 (mTORC2). Thus, DEPTOR knockdown increases the activity of both mTORC1 and mTORC2, thereby promoting cell proliferation and survival. Under certain circumstances, DEPTOR overexpression inhibits S6K1, an mTORC1 substrate, and alleviates S6K-mediated IRS-1/PI3K (phosphoinositide 3 kinase)-dependent negative feedback to activate AKT, leading to cell survival, which facilitates tumor progres$\operatorname{sion}^{2-4}$. Thus, DEPTOR levels need be precisely regulated.

\footnotetext{
Correspondence: Yongchao Zhao (yongchao@zju.edu.cn)

${ }^{1}$ Department of Hepatobiliary and Pancreatic Surgery, the First Affiliated

Hospital, Zhejiang University School of Medicine, Hangzhou, China

${ }^{2}$ Zhejiang Provincial Key Laboratory of Pancreatic Disease, the First Affiliated

Hospital, Zhejiang University School of Medicine, Hangzhou, China

Full list of author information is available at the end of the article

Edited by B. Zhivotovsky
}

ErbB2, also known as Her2 or Neu, is a member of the epidermal growth factor receptor family, which is composed of an extracellular domain, a single transmembrane helix, and a kinase domain ${ }^{5}$. Till date, no ligands for ErbB2 have been identified. Upon stimulation, ErbB2 can only be recruited as a co-receptor to form heterodimers with other members of the ERBB family, such as ErbB1 and ErbB3, and may also form homodimers when it is overexpressed, leading to the phosphorylation and activation of the ErbB2 kinase domain ${ }^{5,6}$. Activated ErbB2 then conveys the extracellular signals to activate intracellular downstream signaling pathways, including the $\mathrm{PI} 3 \mathrm{~K} / \mathrm{AKT} / \mathrm{mTOR}$ pathway and the mitogen-activated protein kinase (MAPK) pathway, leading to cell proliferation, survival, and invasion ${ }^{5}$. ERBB2 gene is overexpressed in $20-30 \%$ of breast cancer cases, which correlates with poor prognosis, lymph-node metastasis, and relative resistance to some drugs ${ }^{6}$. Thus, ErbB2 has served as a biomarker for breast cancer prognosis and a therapeutic target for cancer treatment ${ }^{5}$. Recently, accumulating evidences showed that ErbB2 shuttles into the nucleus and plays important roles in a variety of cellular processes, such as proliferation, signal transduction, and resistance to cancer therapy ${ }^{7-16}$. Mechanistically, ErbB2

\section{(c) The Author(s) 2021}

(c) (i) Open Access This article is licensed under a Creative Commons Attribution 4.0 International License, which permits use, sharing, adaptation, distribution and reproduction cc) in any medium or format, as long as you give appropriate credit to the original author(s) and the source, provide a link to the Creative Commons license, and indicate if changes were made. The images or other third party material in this article are included in the article's Creative Commons license, unless indicated otherwise in a credit line to the material. If material is not included in the article's Creative Commons license and your intended use is not permitted by statutory regulation or exceeds the permitted use, you will need to obtain permission directly from the copyright holder. To view a copy of this license, visit http://creativecommons.org/licenses/by/4.0/. 
contains a putative nuclear localization signal (NLS), which is located adjacent to the transmembrane domain. ErbB2 forms a complex with importin- $\beta$ through the ErbB2-NLS, in which importin- $\beta$ binds to nucleoporins of nuclear pore complexes and leads to ErbB2 nuclear translocation $^{10,11,17}$.

In this study, we report that nuclear ErbB2, acting as a transcription repressor, directly binds to the consensus binding site of the DEPTOR promoter and transcriptionally represses $D E P T O R$ expression, resulting in mTORC1/2 activation. Moreover, ErbB2 activation via autophosphorylation promotes its nuclear translocation and subsequent inhibition of DEPTOR expression to regulate autophagy. Thus, our study uncovered a novel mechanism for ErbB2 in regulation of mTOR signaling and autophagy induction by translocating to the nucleus and directly repressing DEPTOR transcription.

\section{Results}

ErbB2 negatively regulates DEPTOR transcription

ErbB2 is an oncoprotein that promotes cell growth and survival by activating the PI3K/AKT/mTOR and RAS/ ERK pathways ${ }^{5}$. To further determine the mechanism underlying the regulation of these pathways by ErbB2, we transfected two different ErbB2 siRNA oligos into three well-known ErbB2 positive breast cancer cell lines, BT474, SK-BR3, and AU565 (Fig. 1A). Surprisingly, we found a significant accumulation of DEPTOR, a naturally occurring inhibitor of $\mathrm{mTOR}^{2}$ (Fig. 1B). Consistently, ErbB2 silencing reduced the phosphorylation of S6K1 and $\mathrm{AKT}$, the downstream effectors of mTORC1 and mTORC2, respectively, suggesting that ErbB2 knockdown may inhibit the activation of mTORC1 and mTORC2 by DEPTOR induction (Fig. 1B). Further, we found that ErbB2 depletion dramatically increased the mRNA levels of DEPTOR in all the tested cells (Fig. 1C). To exclude the possibility that the increase in mRNA levels of DEPTOR upon ErbB2 knockdown was due to stabilization of DEPTOR mRNA, we simultaneously treated cells with actinomycin D, a RNA synthesis inhibitor ${ }^{18}$, and found that ErbB2 knockdown had no effect on the mRNA halflives of DEPTOR (Fig. 1D). Thus, these results suggest that ErbB2 inactivation promotes the transcription of DEPTOR mRNA, but has no effect on its mRNA stability.

Given that mTORC1 and mTORC2 have been shown to negatively regulate DEPTOR at the mRNA as well as protein levels ${ }^{2}$, we compared the effect of ErbB2 depletion on DEPTOR levels with that of mTOR depletion by silencing $\mathrm{mTOR}$ via two different siRNA oligos. We found that mTOR depletion had a moderate effect on DEPTOR protein levels (Fig. 1E). Consistently, it was observed that mTOR silencing induced DEPTOR transcription (Fig. 1F) by less than two folds in SK-BR3 cells; whereas only slight induction and no induction was observed in AU565 cells and BT474 cells, respectively. Thus, DEPTOR induction by ErbB2 silencing (Fig. 1B, C) was much higher than that by mTOR silencing (Fig. 1E, F), indicating that ErbB2 may directly suppress DEPTOR expression.

\section{ErbB2 translocates to the nucleus and directly binds to DEPTOR promoter to repress its transcription}

It has been previously shown that ErbB2 binds to the consensus Her2-binding site (HAS, HER-2-associated sequence) in the $\mathrm{COX}-2$ promoter and transactivates its transcription in the nucleus ${ }^{7}$. Therefore, we hypothesized that ErbB2 suppresses DEPTOR expression by directly regulating its transcription. We first determined that ErbB2 was indeed located in the nucleus of SK-BR3, BT474, and AU565 cells by immunofluorescence staining using an anti-ErbB2 antibody ${ }^{7,8,14}$ (Fig. 2A). Moreover, ErbB2 was readily detected in the nuclear fractions of SKBR3, BT474, and AU565 cells (Fig. 2B). Next, we performed bioinformatics analysis of the DEPTOR promoter and identified a putative HAS (TCAAATTTC) at -1283 to -1275 , located upstream from the "start" codon of DEPTOR (Fig. 2C). To determine if the HAS plays a role in ErbB2-regulated DEPTOR transcription, we first constructed a luciferase reporter under the control of $D E P$ TOR promoter containing HAS (DEPTOR-Luc). We then performed luciferase reporter assay and found that ErbB2 silencing relieved the inhibition of DEPTOR transcription (Fig. 2C). Next, we constructed another luciferase reporter with the deletion of this putative HAS (DEPTOR-DHAS-Luc) (Fig. 2D), and found that the inhibition of luciferase activity by DEPTOR-Luc was partially abolished by deletion of HAS (Fig. 2D). More importantly, ErbB2 directly bound to the fragment of $D E P T O R$ promoter containing HAS, as demonstrated by chromatin immunoprecipitation (ChIP) assay (Fig. 2E, F). The HAS of COX-2 and another fragment upstream of DEPTOR "start codon" (-6586 to -6449 , DEPTORupstream) were used as positive and negative controls, respectively (Fig. 2E). In addition, we analyzed RNA-seq expression of ErbB2 and DEPTOR in ErbB2-positive BRCA tumor tissues from TCGA. A moderate negative correlation between ErbB2 and DEPTOR $(r=-0.190$, $p=0.042$ ) was found (Fig. 2G). Altogether, our results suggest that nuclear ErbB2 directly binds to the consensus HAS in the DEPTOR promoter and transcriptionally represses DEPTOR expression.

\section{ErbB2 kinase activity is required for its nuclear translocation and transcriptional repression of DEPTOR}

Previous studies showed that inhibiting the kinase activity of ErbB2 or disrupting its nuclear localization suppresses the binding to the promoter of its targeting genes $^{7,19}$. To determine whether the tyrosine kinase activity of ErbB2 is required for its nuclear translocation 


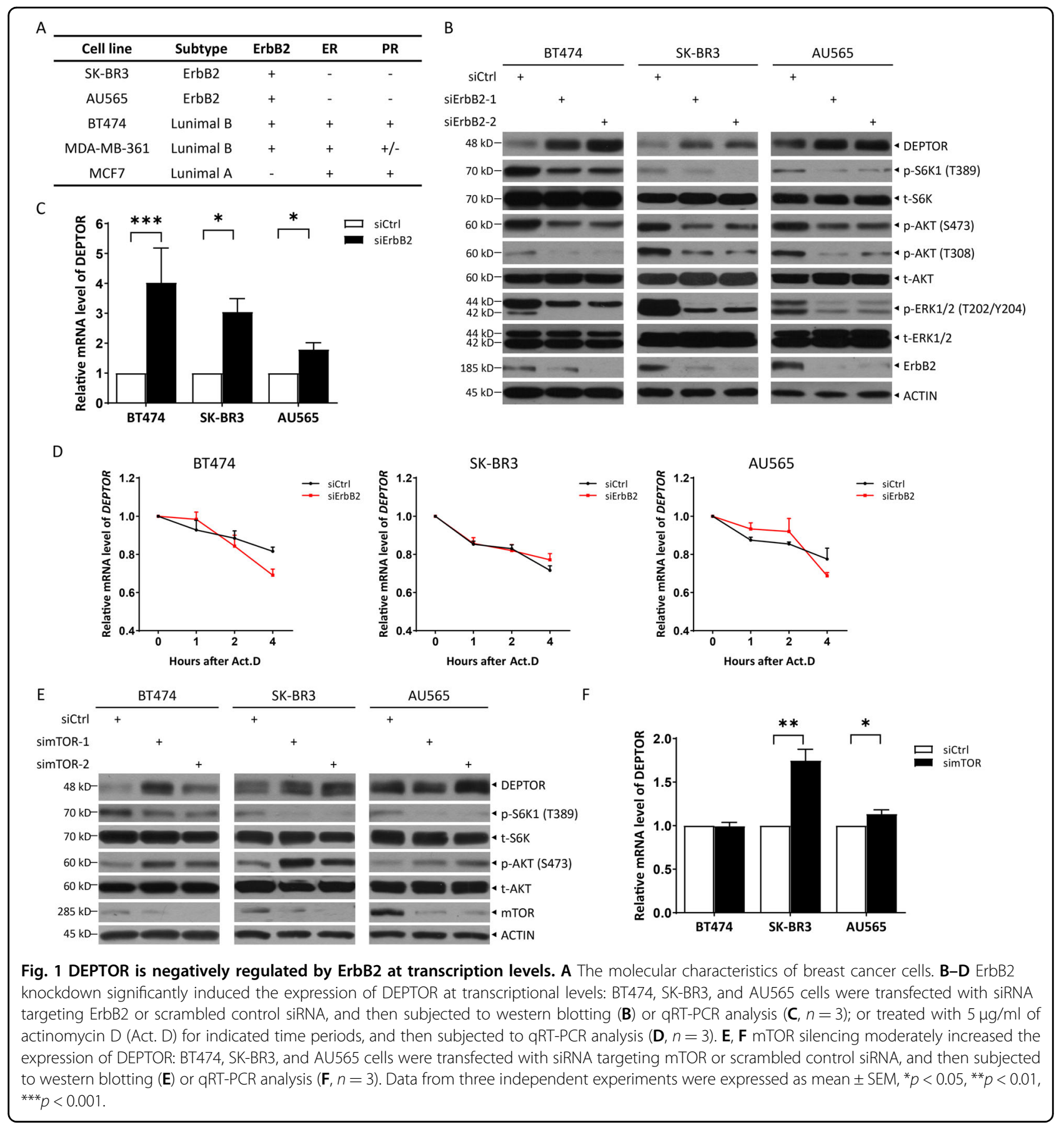

and DEPTOR repression, we treated cells with TAK-165, a specific ErbB2 kinase inhibitor ${ }^{20}$ and found that TAK165 caused a dose- (Fig. 3A) and time-dependent (Fig. 3B) inhibition of ErbB2 autophosphorylation, indicating the inhibition of ErbB2 kinase activity. Importantly, DEPTOR was also induced in a dose- (Fig. 3A) and time-dependent (Fig. 3B) manner upon TAK-165 treatment, suggesting the requirement of ErbB2 kinase activity for DEPTOR repression. We further investigated whether ErbB2 activation promotes its nuclear translocation, and found that TAK-165 treatment suppressed nuclear translocation of ErbB2 (Fig. 3C). Conversely, we overexpressed ErbB2 in ErbB2 relatively low-expressing MCF7 and MDA-MB-361 cells, and found a consequent reduction of DEPTOR (Fig. 3D, lanes 2 vs 1). Moreover, the treatment of HRG $\beta-1$, a ligand binding ErbB3 to stimulate the formation of ErbB2/ ErbB3 dimers and activate ErbB2 ${ }^{13}$, not only decreased DEPTOR significantly in cells transfected with mock 


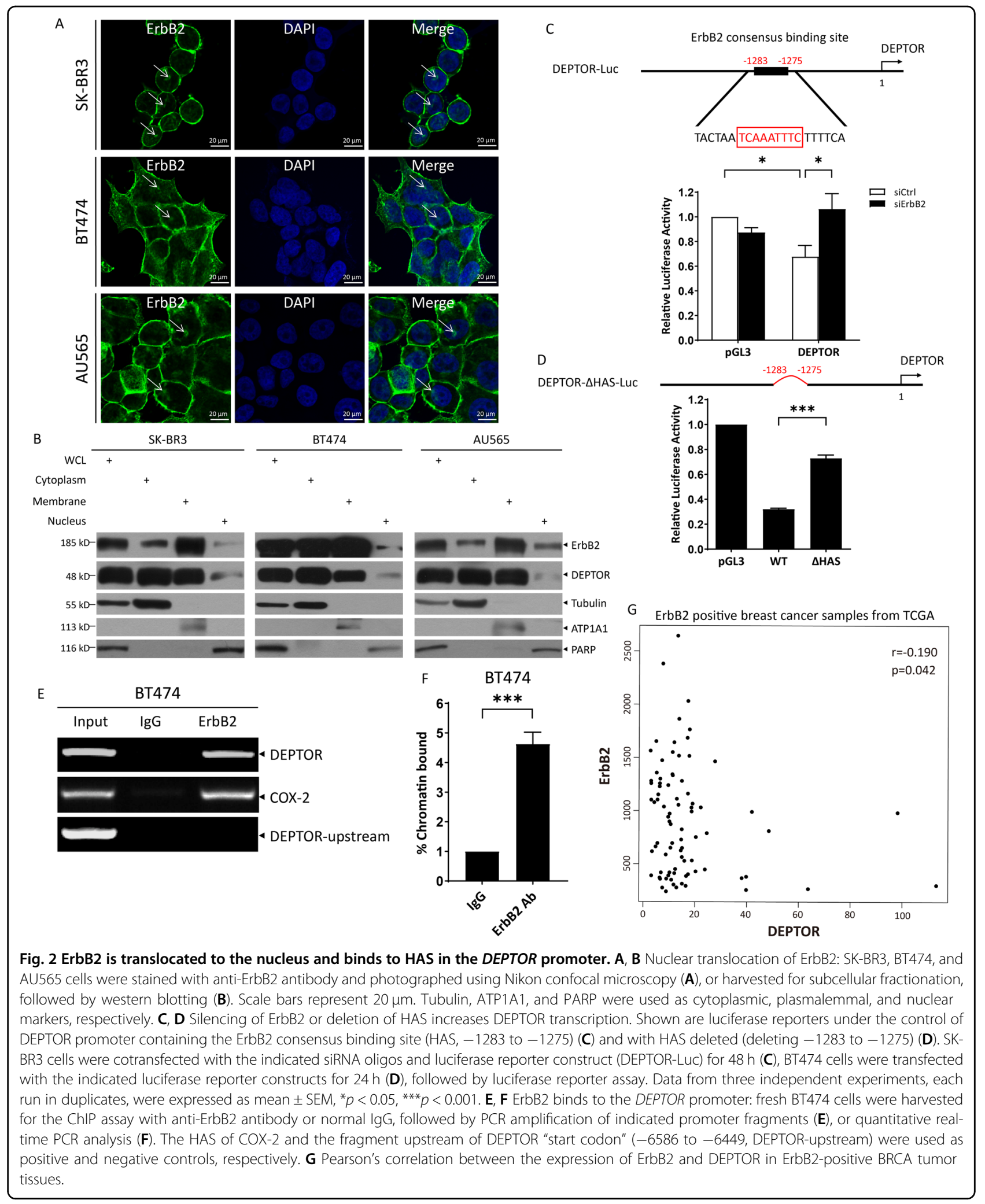

vector (Fig. 3D, lanes 3 vs 1), but also further reduced DEPTOR levels in ErbB2-overexpressed cells (Fig. 3D, LEX, lanes 4 vs 3). Finally, we overexpressed a kinase dead mutant ErbB2 (K753A), and found this mutation impaired the ability to reduce DEPTOR expression regardless of HRG $\beta-1$ treatment (Fig. 3E, lanes 3 vs 2 and 1, lanes 6 vs 5 


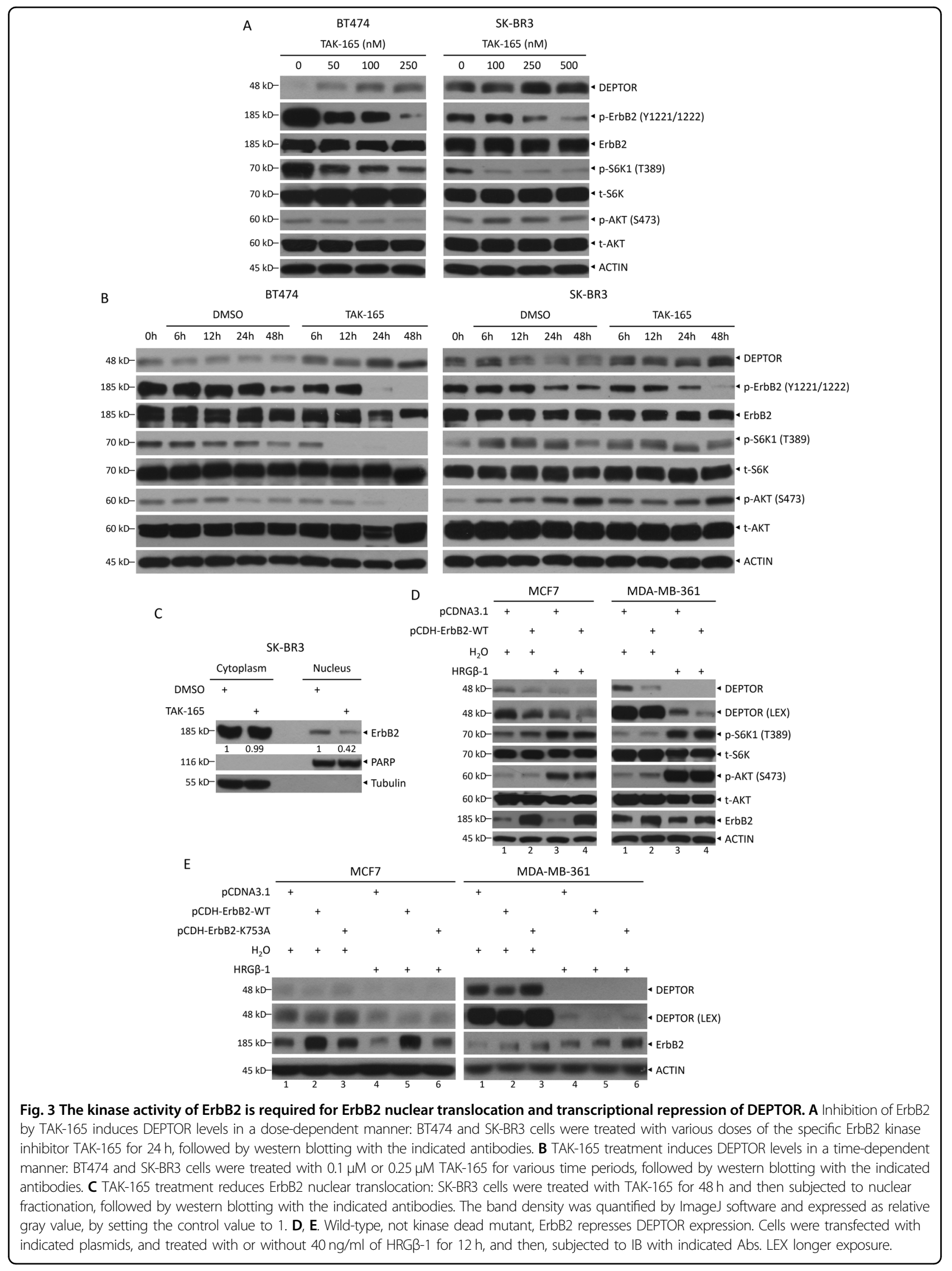


and 4). Altogether, the results suggest that activation of ErbB2 via phosphorylation is critical for the nuclear translocation of ErbB2 and consequent repression of DEPTOR transcription.

\section{ErbB2 inactivation induces autophagy through the induction of DEPTOR}

Several studies clearly demonstrated that DEPTOR induces significant autophagy by mTORC1 inactivation $^{21-28}$. Furthermore, ErbB2 blocks autophagy initiation by directly interacting with Beclin 1, the mammalian orthologue of yeast Atg6 that plays a central role in autophagy ${ }^{29,30}$. Thus, we next determined whether DEPTOR induced by ErbB2 inactivation contributes to autophagy induction upon ErbB2 depletion. Indeed, ErbB2 knockdown effectively induced autophagy, as reflected by the autophagic punctate structures in immunofluorescence staining of endogenous LC3B (Fig. 4A, middle panels), the conversion of LC3-I to LC3II and p62 degradation (Fig. 4B, lanes 2 vs 1), and detectable autophagosomes by electron microscopy (Fig. 4C, middle panels). Meanwhile, DEPTOR was induced significantly along with the inactivation of mTORC1 upon ErbB2 silencing, as reflected by decreased S6K1 phosphorylation (Fig. 4B, lanes 2 vs 1). Interestingly, simultaneous knockdown of DEPTOR partially restored mTORC1 activity (Fig. 4B, lanes 3 vs 2 and 1). More importantly, simultaneous silencing of DEPTOR remarkably reduced autophagic punctate structures (Fig. 4A, right panels), partially inhibited the conversion of LC3-I to LC3-II and p62 degradation (Fig. 4B, lanes 3 vs 2 and 1), and decreased the numbers of autophagosomes (Fig. 4C, right panels), compared to ErbB2 silencing only, indicating a causal role of DEPTOR in autophagy induction by ErbB2 knockdown. Taken together, the results clearly demonstrated that ErbB2 inactivation induces autophagy by inducing DEPTOR to inactivate mTORC1.

\section{Discussion}

In this study, we proved that nuclear ErbB2 represses DEPTOR transcription to effectively block autophagy induction by the following lines of evidence: (1) ErbB2 silencing induced DEPTOR levels at the mRNA as well as protein levels; (2) ErbB2 was readily detected in the nucleus of multiple breast cancer cells, and directly bound to the HAS in the DEPTOR promoter; (3) silencing of ErbB2 or deletion of HAS in the DEPTOR promoter relieved the inhibition of luciferase activity of the $D E P$ TOR promoter; (4) inhibition of ErbB2 kinase activity reduced nuclear translocation of ErbB2, resulting in DEPTOR induction; and (5) ErbB2 silencing induced obvious autophagy, which was partially rescued by simultaneous knockdown of DEPTOR.
It has been previously shown that mTOR negatively regulates DEPTOR at the transcriptional and posttranslational levels ${ }^{2,21,22,31}$. In addition, DEPTOR transcription is positively regulated by Notch1 in T cell leukemogenesis $^{32}$, glucocorticoids in a glucocorticoid receptor-dependent manner during adipogenesis ${ }^{33}$, and Baf60c-Six4 in skeletal muscles ${ }^{34}$. Our study identified the first transcriptional suppressor, ErbB2, which negatively regulates DEPTOR expression. Interestingly, the induction of DEPTOR at protein and mRNA levels by ErbB2 knockdown was much greater than that by mTOR silencing (Fig. 1). Thus, ErbB2 appears to play a significant role in suppressing DEPTOR transcription in ErbB2 positive breast cancer cells.

Previous studies have shown that ErbB2 is located in the nucleus and acts as a transcription regulator to activate the transcription of $\mathrm{COX}-2$ by directly binding to the HAS in its promoter ${ }^{7}$, or to enhance rRNA synthesis by promoting the binding of RNA Pol I to rDNA $^{8}$, etc. Further studies revealed that nuclear ErbB2 is involved in breast cancer cell growth, metastasis, and drug resistance, and it is being validated as a novel therapeutic target in ErbB2positive breast cancer $^{11}$. In this study, we report that nuclear ErbB2 serves as a transcriptional suppressor, as evidenced by the enhancement in transcriptional activity of the DEPTOR promoter upon ErbB2 silencing (Fig. 2C) or deletion of HAS (Fig. 2D). This transcription suppressing activity might be determined by the interaction of ErbB2 with certain proteins in the nucleus, which needs to be further explored. Likewise, the transcriptional regulation of both $D E P T O R$ and $C O X-2$ requires the kinase activity of ErbB2. It has been previously shown that functional ErbB2 is required for binding to the $\mathrm{COX}-2$ promoter $^{7}$. In our study, inhibition of ErbB2 by its specific inhibitor TAK-165 reduced ErbB2 levels in the nucleus (Fig. 3C), but increased DEPTOR levels (Fig. 3A, B). These results implied that the increase in DEPTOR levels upon TAK-165 treatment might be attributed to the decrease in ErbB2 nuclear translocation (Fig. 3C). Furthermore, ErbB2 inactivation induced DEPTOR to inhibit mTORC1, subsequently leading to autophagy induction (Fig. 4). Thus, the inhibition of mTOR by DEPTOR may contribute to the therapeutic role of targeting nuclear ErbB2.

In addition, previous studies have shown that ErbB2 can block autophagy initiation by modulation of Beclin 1 in breast cancer and Alzheimer's disease $\mathrm{e}^{29,30}$, and regulate autophagic cell death by modulation of ATG4B expression in retinal pigment epithelium cells ${ }^{35}$. The incidence of mammary tumors triggered by mammary-specific overexpressing activated ErbB2 was obviously lower in mice carrying a knock-in mutant Becn1 $1^{\mathrm{F} 121 \mathrm{~A} / \mathrm{F} 121 \mathrm{~A}}$ with increased autophagy, compared to Becn $1^{\mathrm{WT} / \mathrm{WT}}$ mice, and these mutant Becn1 $1^{\mathrm{F} 121 \mathrm{~A} / \mathrm{F} 121 \mathrm{~A}}$ mice had significant longer life span, demonstrating that ErbB2-mediated autophagy 


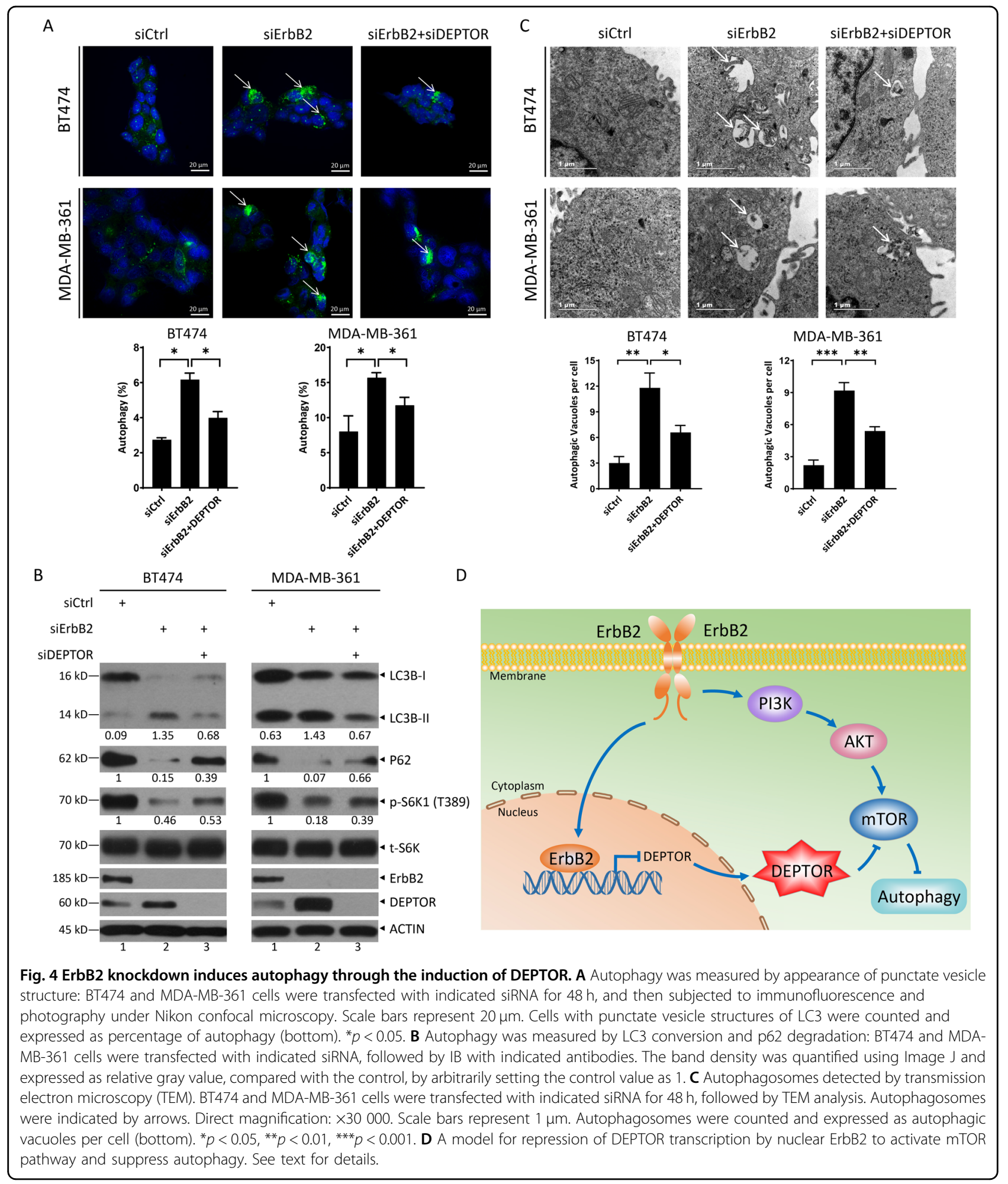

suppression facilitates breast tumorigenesis ${ }^{30}$. Interestingly, ErbB2-mediated autophagy suppression also renders ErbB2-induced breast tumorigenesis in a Beclin 1independent manner ${ }^{36}$. It is worth to investigate whether ErbB2-induced breast tumorigenesis is mediated by autophagy suppression via DEPTOR repression. Moreover, an autophagy-inducing peptide totally suppressed the growth of ErbB2-positive xenografts ${ }^{30}$, implying that targeting ErbB2-mediated autophagy suppression might represent an attractive approach for ErbB2-positive breast 
cancer therapy. Consistently, the induced autophagy contributes to the efficacy of ErbB2-targeted therapies ${ }^{37}$, enhancing tumor cells killing ${ }^{38}$, paradoxically, or facilitating drug resistance ${ }^{39,40}$. The complex roles of autophagy in ErbB2-targeted therapies warrant further investigation. In our study, we found that ErbB2 inactivation dramatically induced autophagy by inducing DEPTOR in ErbB2-positive breast cancer cells (Figs. 1B, $3 \mathrm{~A}, \mathrm{~B}, 4 \mathrm{~A}, \mathrm{~B})$. Thus, whether and how autophagy regulated by the ErbB2-DEPTOR axis contributes to ErbB2targeted therapies is an interesting topic to further explore using mouse model of breast cancer. Finally, given that DEPTOR is a direct inhibitor of mTORC1 and mTORC2 ${ }^{4}$, induced DEPTOR upon ErbB2 inactivation not only promoted autophagy, but also might mediate cell proliferation, survival, and drug resistance ${ }^{21,22}$. Thus, targeting DEPTOR may be an attractive approach to overcome the resistance of ErbB2-targeted therapies.

In summary, our study identified the mTOR inhibitor, DEPTOR, as a novel downstream target of nuclear ErbB2. In addition to activation of the PI3K/AKT/mTOR pathway in a classical way in response to extracellular signals, ErbB2 is translocated to the nucleus, where it represses DEPTOR transcription to further activate the PI3K/AKT/ mTOR pathway and suppress autophagy, which adds another layer of complexity for ErbB2 to regulate the mTOR pathway, leading to autophagy (Fig. 4D).

\section{Methods}

\section{Cell culture and chemicals}

SK-BR3, BT474, AU565, MCF7, and MDA-MB-361 cells were obtained from American Type Culture Collection (ATCC). All the cell lines were authenticated by the ATCC, and were expanded and preserved in liquid nitrogen upon receipt. Cells for experiments were passaged for fewer than 25-30 times. SK-BR3, MCF7, and MDA-MB-361 cells were maintained in Dulbecco's modified Eagle medium (DMEM) supplemented with 10\% $(\mathrm{v} / \mathrm{v})$ fetal bovine serum (FBS) and $1 \%(\mathrm{v} / \mathrm{v})$ penicillinstreptomycin (PS) at $37^{\circ} \mathrm{C}$ in a $5 \% \mathrm{CO}_{2}$ humidified incubator. BT474 and AU565 cells were maintained in Roswell Park Memorial Institute (RPMI) 1640 medium supplemented with 10\% FBS and 1\% PS. Mubritinib (TAK-165, HY-13501) and HRGß1 (HY-P7365) were purchased from MedChem Express.

\section{Western blotting}

Cells were lysed in lysis buffer in the presence of protease inhibitors and phosphatase inhibitors. Cell lysates were then subjected to western blotting as previously described $^{41}$. Primary antibodies were used as follows: $\mathrm{p}$ ErbB2 (Y1221/1222) (2243\#), ErbB2 (2165\#), DEPTOR (11816\#), p-AKT (S473) (4060\#), p-AKT (T308) (4056\#), t-AKT (4691\#), p-S6K1 (T389) (9234\#), p-ERK (T202/
Y204) (9101\#), t-ERK (4696\#), mTOR (2972\#), PARP (9532\#), and LC3B (2775\#) (Cell Signaling Technology); tS6K (sc-230\#), and ErbB2 (sc-33684\#) (Santa Cruz); Tubulin (T9026\#), LC3B (L7543\#), and ACTIN (A5441\#) (Sigma); p62 (PM045\#) (Medical \& Biological Laboratories).

\section{siRNA transfection}

Cells were transfected with siRNA oligos in 60-mm dishes or 6-well plates using Lipofectamine 2000, according to the manufacturer's instructions (Invitrogen). The sequences of siRNA oligonucleotides were as follows: siErbB2-1: $5^{\prime}$-GCA GTTACCAGTGCCAATA-3'; siErbB2-2: 5'-AAATTCCAG TGGCCATCAA-3'; simTOR-1: 5'-AAGAATCAAAGAGC AGAGTGC-3'; simTOR-2: 5'-GCTGTGCTACACTACAA ACAT-3'; and siCtrl: 5'-ATTGTATGCGATCGCAGAC-3'.

\section{DNA transfection and dual-luciferase assay}

The luciferase reporter driven by DEPTOR promoter was generated by PCR amplification using pfx DNA polymerase (Invitrogen) with the following primers: DEPTOR-luc-F: 5'-AGATCTGGTACCGAGGATAAAG TGTTTGGCACAATGT-3' and DEPTOR-luc-R: $5^{\prime}$-AGA TCTCTCGAGGCTGTAAGCCGAGTTCGGGT- $3^{\prime}$. The PCR products were subcloned into the KpnI and XhoI sites of pGL3 luciferase reporter, subsequently verified by Sanger sequencing. Cells were transfected with $1 \mu \mathrm{g}$ of luciferase reporter constructs, along with $0.2 \mu \mathrm{g}$ of Renilla construct, using Lipofectamine 3000 following the manufacturer's instructions (Invitrogen). Luciferase activity was measured using the Promega Dual-Luciferase Reporter Assay System kit (E1910, Promega), following the manufacturer's instructions. The relative firefly luciferase activity was normalized to Renilla luciferase activity.

\section{Immunofluorescence staining}

For immunofluorescence staining, the cells were first fixed with $4 \%$ formaldehyde for $15 \mathrm{~min}$ and then treated with $0.05 \%$ TritonX-100 for $10 \mathrm{~min}$. Next, the cells were blocked for $30 \mathrm{~min}$, and stained with anti-ErbB2 antibody (1:500) or anti-LC3B antibody (1:500, Sigma) for $1 \mathrm{~h}$, followed by staining with secondary antibodies conjugated with Alexa Fluor 488 (1:500, Abcam) for $30 \mathrm{~min}$ and DAPI (1:1000, Beyotime) for $10 \mathrm{~min}$ at room temperature. The cells were then photographed under a confocal fluorescence microscope (Nikon).

\section{Subcellular fractionation}

Cell fractions were extracted using Cell Fractionation Kit (9038\#, Cell Signaling Technology), according to the manufacturer's instructions. Briefly, cells harvested by trypsin were lysed in cytoplasm isolation buffer containing protease inhibitors and $1 \mathrm{mM}$ PMSF, vortexed for $5 \mathrm{~s}$ at 
ultrahigh speed to fully resuspend, and then incubated on ice for $5 \mathrm{~min}$, followed by centrifugation at $500 \mathrm{~g}$ for $5 \mathrm{~min}$ at $4{ }^{\circ} \mathrm{C}$. The supernatants were transferred to a clean tube as cytoplasmic fractions. The pellets were lysed in membrane isolation buffer containing protease inhibitors and $1 \mathrm{mM}$ PMSF, vortexed for $15 \mathrm{~s}$ at ultrahigh speed to fully resuspend, and then incubated on ice for $5 \mathrm{~min}$, followed by centrifugation at $8000 \mathrm{~g}$ for $5 \mathrm{~min}$ at $4{ }^{\circ} \mathrm{C}$. The supernatants were saved as membrane and organelle fractions. The pellets were ultrasound lysed in cytoskeleton/nucleus isolation buffer containing protease inhibitors and $1 \mathrm{mM}$ PMSF, at $60 \%$ of power for $5 \mathrm{~min}$ at $4{ }^{\circ} \mathrm{C}$, followed by centrifugation at $13,600 \mathrm{rpm}$ for $5 \mathrm{~min}$ at $4{ }^{\circ} \mathrm{C}$. The supernatants were collected as cytoskeletal and nuclear fractions.

\section{Chromatin immunoprecipitation (ChIP)}

The ChIP assay was performed using the Simple ChIP Enzymatic Chromatin IP Kit (9003\#, Cell Signaling Technology) according to the manufacturer's instructions. The primer sequences for DEPTOR and COX-2 were as follows: DEPTOR-F: 5'-ATACTGCCATAAACATTACT TCGCC- $3^{\prime}$ and DEPTOR-R: 5'-GGTATTGTCTAT CCG TAAAAGATTATGAA-3'; COX-2-F: 5'-CTTCAAAATA AGCTTGAATTCAGGATTGTAATG- ${ }^{\prime}$ and COX-2-R: 5'-CTTTTTGATAATTTAATAATTTCAATCTTCTGT TTC-3'; DEPTOR-upstream-F: 5'-AGGAGACCTACAA GCATTTCGTG-3' and DEPTOR-upstream-R: $5^{\prime}$-TTCA TTTCCAACCCTGCTCAC-3'.

\section{Quantitative RT-PCR}

Quantitative RT-PCR analysis was performed as described previously ${ }^{42}$. Briefly, total RNA was extracted from cells using TRIzol reagent (15596018, Invitrogen). cDNA was synthesized from RNA using the PrimeScript RT reagent kit (RR037A, Takara). Quantitative real-time PCR was accomplished using SYBR Premix Ex Taq (RR420A, TaKaRa) on an Applied Biosystems StepOnePlus ${ }^{\text {TM }}$ Real-Time PCR instrument. The primer sequences were as follows: DEPTOR-F: 5'-GCAGCAGGAAT GAAGGTCTG-3' ${ }^{\prime}$ and DEPTOR-R: ${ }^{\prime}$-GTATGTGCGGA GAAGACTCGTAT-3'; GAPDH-F: 5'-AGGGCATCCTG GGCTACAC-3' and GAPDH-R: $5^{\prime}$-GCCAAATTCGTTG TCAT ACCAG-3'.

\section{Transmission electron microscopy}

BT474 and MDA-MB-361 cells cultured in 60-mm dishs were collected by trypsin. Cells were rinsed with $0.1 \mathrm{M}$ phosphate buffer ( $\mathrm{pH}$ 7.4) before fixing with $2.5 \%$ glutaraldehyde in phosphate buffer at $4{ }^{\circ} \mathrm{C}$ overnight, and then post-fixed in $1 \%$ Osmic acid at room temperature for 1-2 h. After ethanol and acetone dehydration, penetrant treating and embedding in polybed 812 resin, thin sections $(70 \mathrm{~nm})$ were post-stained with $2 \%$ uranyl acetate followed by $0.3 \%$ lead citrate for $10 \mathrm{~min}$. The photos of sample sections were taken using a TECNAI 10 transmission electron microscope (FEI Company, Hillsboro, OR) at $120 \mathrm{kV}$. To quantify autophagic vacuoles, five micrographs were taken with systematic random sampling from each sample.

\section{Correlation between the expression of ErbB2 and DEPTOR in breast cancer}

Gene-level expression data (in format of FPKM) of ErbB2 and DEPTOR from the TCGA-BRCA project in The Cancer Genome Atlas (TCGA) were downloaded from the GDC data portal (https://portal.gdc.cancer.gov/). FPKM refers to Fragments Per Kilobase of transcript per Million mapped reads. A total of 83 ErbB2-positive BRCA tumor samples with FPKM of ErbB2 over 200 were included in the analysis. Pearson's correlation between the expression of ErbB2 and DEPTOR was calculated in these 83 BRCA tumor samples.

\section{Statistical analysis}

The data from three independent experiments were expressed as the mean \pm SEM and analyzed using GraphPad Prism 5. The comparison of parameters between groups was performed using the two-tailed Student's $t$-test with SPSS 20.0 (IBM). $p<0.05$ was considered statistically significant.

Acknowledgements

The authors would like to thank Dr. Han Liu for the pCDH-ErbB2 plasmid.

\section{Author details}

'Department of Hepatobiliary and Pancreatic Surgery, the First Affiliated Hospital, Zhejiang University School of Medicine, Hangzhou, China. ${ }^{2}$ Zhejiang Provincial Key Laboratory of Pancreatic Disease, the First Affiliated Hospital, Zhejiang University School of Medicine, Hangzhou, China. ${ }^{3}$ Institute of Translational Medicine, Zhejiang University School of Medicine, Hangzhou, China. ${ }^{4}$ Cancer Institute of the Second Affiliated Hospital, Zhejiang University School of Medicine, Hangzhou, China. ${ }^{5}$ Cancer Center, Zhejiang University, Hangzhou, China

\section{Author contributions}

Y.B. designed and performed the experiments, analyzed and interpreted the data, and drafted the manuscript. L.G. revised the manuscript. P.L. designed and performed the experiments. X.X. analyzed and interpreted the data and revised the manuscript. Y.Z. designed the study, analyzed and interpreted the data, and revised and finalized the manuscript. All authors have reviewed the manuscript.

\section{Funding}

This work was supported by the National Key R\&D Program of China (2016YFA0501800 to Y.Z. and X.X.), the National Natural Science Foundation of China $(81672728,81972591$, and 92053117 to Y.Z., 81572708 and 81974429 to X.X.), and the Natural Science Foundation of Zhejiang Province (LR16C050001 to $Y . Z$.).

Conflict of interest

The authors declare no competing interests. 


\section{Publisher's note}

Springer Nature remains neutral with regard to jurisdictional claims in published maps and institutional affiliations.

Received: 2 November 2020 Revised: 25 March 2021 Accepted: 30 March 2021

\section{Published online: 14 April 2021}

\section{References}

1. Zoncu, R., Efeyan, A. \& Sabatini, D. M. mTOR: from growth signal integration to cancer, diabetes and ageing. Nat. Rev. Mol. Cell Biol. 12, 21-35 (2011).

2. Peterson, T. R. et al. DEPTOR is an mTOR inhibitor frequently overexpressed in multiple myeloma cells and required for their survival. Cell 137, 873-886 (2009).

3. Zhao, Y. \& Sun, Y. Targeting the mTOR-DEPTOR pathway by CRL E3 ubiquitin ligases: therapeutic application. Neoplasia 14, 360-367 (2012).

4. Caron, A., Briscoe, D. M., Richard, D. \& Laplante, M. DEPTOR at the nexus of cancer, metabolism, and immunity. Physiol. Rev. 98, 1765-1803 (2018).

5. Yarden, Y. \& Pines, G. The ERBB network: at last, cancer therapy meets systems biology. Nat. Rev. Cancer 12, 553-563 (2012).

6. Harari, D. \& Yarden, Y. Molecular mechanisms underlying ErbB2/HER2 action in breast cancer. Oncogene 19, 6102-6114 (2000).

7. Wang, S. C. et al. Binding at and transactivation of the COX-2 promoter by nuclear tyrosine kinase receptor ErbB-2. Cancer Cell 6, 251-261 (2004).

8. Li, L. Y. et al. Nuclear ErbB2 enhances translation and cell growth by activating transcription of ribosomal RNA genes. Cancer Res. 71, 4269-4279 (2011).

9. Wang, S. C. \& Hung, M. C. Nuclear translocation of the epidermal growth factor receptor family membrane tyrosine kinase receptors. Clin. Cancer Res. 15, 6484-6489 (2009).

10. Wang, Y. N. \& Hung, M. C. Nuclear functions and subcellular trafficking mechanisms of the epidermal growth factor receptor family. Cell Biosci. 2, 13 (2012).

11. Cordo Russo, R. I., Chervo, M. F., Madera, S., Charreau, E. H. \& Elizalde, P. V. Nuclear ErbB-2: a novel therapeutic target in ErbB-2-positive breast cancer? Horm. Cancer 10, 64-70 (2019).

12. Schillaci, R. et al. Clinical relevance of ErbB-2/HER2 nuclear expression in breast cancer. BMC Cancer 12, 74 (2012).

13. Elizalde, P. V., Cordo Russo, R. I., Chervo, M. F. \& Schillaci, R. ErbB-2 nuclear function in breast cancer growth, metastasis and resistance to therapy. Endocr. Relat. Cancer 23, T243-T257 (2016).

14. Cordo Russo, R. I. et al. Targeting ErbB-2 nuclear localization and function inhibits breast cancer growth and overcomes trastuzumab resistance. Oncogene 34, 3413-3428 (2015).

15. Venturutti, L. et al. Stat3 regulates ErbB-2 expression and co-opts ErbB-2 nuclear function to induce miR-21 expression, PDCD4 downregulation and breast cancer metastasis. Oncogene 35, 2208-2222 (2016).

16. Beguelin, W. et al. Progesterone receptor induces ErbB-2 nuclear translocation to promote breast cancer growth via a novel transcriptional effect: ErbB-2 function as a coactivator of Stat3. Mol. Cell Biol. 30, 5456-5472 (2010).

17. Giri, D. K. et al. Endosomal transport of ErbB-2: mechanism for nuclear entry of the cell surface receptor. Mol. Cell Biol. 25, 11005-11018 (2005).

18. Perry, R. P. \& Kelley, D. E. Inhibition of RNA synthesis by actinomycin D: characteristic dose-response of different RNA species. J. Cell Physiol. 76, 127-139 (1970)

19. Diaz Flaque, M. C. et al. Progestin drives breast cancer growth by inducing p21 (CIP1) expression through the assembly of a transcriptional complex among Stat3, progesterone receptor and ErbB-2. Steroids 78, 559-567 (2013).
20. Lin, N. U. \& Winer, E. P. New targets for therapy in breast cancer: small molecule tyrosine kinase inhibitors. Breast Cancer Res. 6, 204-210 (2004).

21. Zhao, Y., Xiong, X. \& Sun, Y. DEPTOR, an mTOR inhibitor, is a physiological substrate of SCF(betaTrCP) E3 ubiquitin ligase and regulates survival and autophagy. Mol. Cell 44, 304-316 (2011)

22. Gao, D. et al. mTOR drives its own activation via SCF(betaTrCP)dependent degradation of the mTOR inhibitor DEPTOR. Mol. Cell 44, 290-303 (2011).

23. Antonioli, M. et al. AMBRA1 interplay with cullin E3 ubiquitin ligases regulates autophagy dynamics. Dev. Cell 31, 734-746 (2014).

24. Zhao, Y., Xiong, X., Jia, L. \& Sun, Y. Targeting Cullin-RING ligases by MLN4924 induces autophagy via modulating the HIF1-REDD1-TSC1-mTORC1-DEPTOR axis. Cell Death Dis. 3, e386 (2012).

25. Luo, Z. et al. The Nedd8-activating enzyme inhibitor MLN4924 induces autophagy and apoptosis to suppress liver cancer cell growth. Cancer Res. 72 , 3360-3371 (2012).

26. Zhao, Y. \& Sun, Y. Cullin-RING ligases as attractive anti-cancer targets. Curr. Pharm. Des. 19, 3215-3225 (2013).

27. Zhao, Y., Morgan, M. A. \& Sun, Y. Targeting Neddylation pathways to inactivate cullin-RING ligases for anticancer therapy. Antioxid. Redox Signal. 21, 2383-2400 (2014).

28. Xiong, $X$. et al. Ribosomal protein S27-like regulates autophagy via the betaTrCP-DEPTOR-mTORC1 axis. Cell Death Dis. 9, 1131 (2018).

29. Wang, B. J. et al. ErbB2 regulates autophagic flux to modulate the proteostasis of APP-CTFs in Alzheimer's disease. Proc. Natl Acad. Sci. USA 114, E3129-E3138 (2017).

30. Vega-Rubin-de-Celis, S. et al. Increased autophagy blocks HER2-mediated breast tumorigenesis. Proc. Natl Acad. Sci. USA 115, 4176-4181 (2018).

31. Duan, $\mathrm{S}$. et al. mTOR generates an auto-amplification loop by triggering the betaTrCP- and CK1alpha-dependent degradation of DEPTOR. Mol. Cell $\mathbf{4 4}$ 317-324 (2011).

32. $\mathrm{Hu}$, Y. et al. DEPTOR is a direct NOTCH1 target that promotes cell proliferation and survival in T-cell leukemia. Oncogene 36, 1038-1047 (2017).

33. Laplante, M. et al. DEPTOR cell-autonomously promotes adipogenesis, and Its expression is associated with obesity. Cell Metab. 16, 202-212 (2012).

34. Meng, Z.-X. et al. Baf60c drives glycolytic metabolism in the muscle and improves systemic glucose homeostasis through Deptor-mediated Akt activation. Nat. Med. 19, 6 (2013).

35. Sheu, S. J., Chen, J. L., Bee, Y. S., Lin, S. H. \& Shu, C. W. ERBB2-modulated ATG4B and autophagic cell death in human ARPE19 during oxidative stress. PLOS ONE 14, e0213932 (2019).

36. Lozy, F. et al. ERBB2 overexpression suppresses stress-induced autophagy and renders ERBB2-induced mammary tumorigenesis independent of monoallelic Becn1 loss. Autophagy 10, 662-676 (2014).

37. Janser, F. A., Tschan, M. P. \& Langer, R. The role of autophagy in HER2-targeted therapy. Swiss Med. Wkly. 149, w20138 (2019).

38. Zhu, X. et al. Autophagy stimulates apoptosis in HER2-overexpressing breast cancers treated by lapatinib. J. Cell Biochem. 114, 2643-2653 (2013).

39. Chen, S. et al. Autophagy facilitates the Lapatinib resistance of HER2 positive breast cancer cells. Med. Hypotheses 77, 206-208 (2011).

40. Rodriguez, C. E., Reidel, S. I., Bal de Kier Joffe, E. D., Jasnis, M. A. \& Fiszman, G. L. Autophagy protects from trastuzumab-induced cytotoxicity in HER2 overexpressing breast tumor spheroids. PLOS ONE 10, e0137920 (2015).

41. Cui, D. et al. The cross talk of two family members of beta-TrCP in the regulation of cell autophagy and growth. Cell Death Differ. 27, 1119-1133 (2020).

42. Cui, D. et al. FBXW7 confers radiation survival by targeting p53 for degradation. Cell Rep. 30, 497-509 e494 (2020). 